\title{
Jakob L. FINK (éd.), The Development of Dialectic from Plato to Aristotle
}

Juliette Lemaire

\section{OpenEdition}

\section{Journals}

Édition électronique

URL : https://journals.openedition.org/philosant/456

DOI : $10.4000 /$ philosant.456

ISSN : 2648-2789

\section{Éditeur}

Éditions Vrin

\section{Édition imprimée}

Date de publication : 24 novembre 2015

Pagination : 265-269

ISBN : 978-2-7574-1141-4

ISSN : $1634-4561$

Référence électronique

Juliette Lemaire, « Jakob L. Fink (éd.), The Development of Dialectic from Plato to Aristotle », Philosophie antique [En ligne], 15 | 2015, mis en ligne le 01 novembre 2018, consulté le 03 décembre 2022. URL : http://journals.openedition.org/philosant/456 ; DOI : https://doi.org/10.4000/philosant.456

\section{(c) (†) $\ominus$}

Creative Commons - Attribution - Pas d'Utilisation Commerciale - Pas de Modification 4.0 International - CC BY-NC-ND 4.0

https://creativecommons.org/licenses/by-nc-nd/4.0/ 
comment la rhétorique s'y prendra-t-elle pour identifier avec sûreté la catégorie psychique dont relève tel ou tel citoyen qui doit être persuadé par une forme déterminée de discours ? Difficulté qui revient d'une autre manière quand il faudra discerner empiriquement les tempéraments (les modérés ou les ardents) et éliminer les enfants non éducables (308e-309b). Le politique tisse un matériau qu'il a luimême choisi ( $c f$. p. 274 in fine) : quel savoir dirigera avec autorité et rigueur son doigt sélectif ? Il est introuvable dans le cadre de l'épistémologie générale de Platon. L'Étranger le dit à sa manière, sur un autre plan : la politique est aussi connaissance du kairos, du moment opportun qui permettra de dire si oui ou non il faut recourir à la rhétorique, à la stratégie ou à l'art judiciaire, autrement dit, s'il faut (ou non) persuader, faire la guerre, lancer un procès (305d). Ce kairos est éminemment changeant, il n'obéit à aucune loi, il n'a pas de critère assuré - il est cette fenêtre qu'ouvre provisoirement le devenir à une action susceptible d'orienter son cours dans une certaine direction. Mais, du kairos (comme d'ailleurs du choix des caractères), il n'y a pas de science, car il n'y a de science que de ce qui est toujours identique à soi, de ce qui, précisément, est hors du devenir.

Peut-on réduire la contradiction entre l'épistémologie générale de Platon et sa conception particulière de la science politique dans notre dialogue ? La question reste en suspens. À moins, peut-être, que cette science ne soit seulement celle des bonnes intentions : viser le bien, préférer la limite à l'illimité, introduire la juste mesure, ce qui passerait par l'institution d'une orthe doxa et l'assortiment réglé des tempéraments contraires. Admettons. Mais en l'absence d'un modus operandi déterminé, il s'agit là d'une science terriblement formelle et vague : l'exigence de détermination et de mesure manque à son tour de détermination et de mesure - et s'avère incapable d'asseoir la légitimité du politique.

Quoi qu'il en soit de cette difficulté sans doute insoluble, l'ouvrage de Dimitri El Murr demeure pour qui veut comprendre le Politique, et plus largement la pensée platonicienne, une référence obligatoire, tant par la finesse de ses analyses et la clarté de son écriture que par les questions qu'il invite à poser.

Fulcran TEISSERENC

GRAMATA, Université Paris I Panthéon-Sorbonne

Jakob L. FINK (éd.), The Development of Dialectic from Plato to Aristotle, VII355p., Cambridge-New York, Cambridge University Press, 2012, ISBN 978-1107-01222-6

Actes d'un colloque qui s'est tenu à Copenhague en 2007, l'ouvrage veut combler un manque : traiter du développement de la dialectique de Platon à Aristote, non pas en se focalisant sur la méthode et l'ontologie, mais en se fondant sur le cadre du débat dialectique. Selon J. Fink (p. 2), la dialectique ici signifie d'abord 
et avant tout argumentation adressée à un interlocuteur. La pratique de l'argumentation dialectique et son extension dans la forme littéraire du dialogue constituent le cœur de l'ouvrage. Que devient le dialegesthai de Socrate à Aristote ? En tant qu'héritiers de Socrate, Platon et Aristote ont une conception de la dialectique qui se prête à la comparaison. Le schéma visible sur la couverture et page 3 pose le cadre : questionneur, répondant, relation (égale ou inégale) entre eux, le sujet discuté, le caractère du répondant, le public, l'usage de l'argument dialectique - certains de ces éléments se trouvent chez Platon, mais c'est Aristote qui les énonce tous. Ne court-on pas le risque de mésinterpréter la dialectique de Platon en partant de ce qu'énonce Aristote dans les Topiques et les Réfutations sophistiques ? Non, car Aristote ne fait qu'expliciter ce qui est déjà implicitement présent chez Platon. La conception aristotélicienne de la dialectique sert ainsi d'instrument heuristique. Le dipositif questionneur/répondant est le même, de Platon à Aristote, mais le type de question change. La question « qu'est-ce que $\mathrm{X}$ ? » est la question socratique par excellence, tandis que pour Aristote, la question dialectique appelle une réponse seulement par oui ou non. Cela indique certes un changement dans la conception de la dialectique, mais cela ne sape pas le fondement commun de la dialectique chez Platon et Aristote. Étudier le développement de la dialectique signifie étudier la forme et le contenu du dialogue. Mais en quel sens entendre « développement » ici ? Il ne s'agit pas de suivre un seul schéma de développement soit chez Aristote, soit chez Platon, ou bien de tenter d'avoir une lecture évolutionniste à la manière de Jaeger. Une autre possibilité serait l'harmonisation néoplatonicienne. Mais celle-ci fait fi de l'aspect dialogique et de la forme questions/réponses et lit Platon sans considérer Socrate.

Les articles sont regroupés en trois thèmes: la dialectique comme activité interpersonnelle; forme et contenu dans le dialogue philosophique; la méthodologie dialectique. Tous les aspects de la dialectique ne sont pas traités, en particulier la dialectique platonicienne telle qu'on la rencontre dans le Phédon et la République. Mais sur ces questions, de nombreuses études existent déjà, alors que les aspects traités ici sont la plupart du temps négligés - par exemple le rôle du répondant.

La contribution de L. Castagnoli montre par l'analyse détaillée de quatre arguments, (Platon, Euthydème, 286c-288a ; Thététe, 169e-171c ; Aristote, Métaphysique, $\Gamma 8,1012 \mathrm{~b} 13-18$, l'argument protreptique) qu'il n'est pas possible d'interpréter les arguments d'autoréfutation en les dépouillant de leurs vêtements dialogiques, sous peine de leur faire perdre leur force logique ou philosophique. Ainsi de Théétète, 171a, qui ne peut être compris comme une consequentia miserabilis, c'est-à-dire la loi selon laquelle toute proposition qui implique sa propre contradictoire est nécessairement fausse. En effet, on ne trouve aucune formulation de cette loi dans les textes anciens. L. Castagnoli a le mérite de rappeler ce principe exégétique : les lois de notre «logique classique » n’ont pas besoin de coïncider avec celles de la logique ancienne. La consequentia miserabilis ne 
sous-tend aucun des quatre arguments d'autoréfutation analysés ici. Loin de signaler une déficience logique, cela montre plutôt que la reconstruction hors du contexte dialectique de ce type d'argumentation est logiquement suspecte. Le contexte dialectique n'est pas qu'un cadre mais la condition nécesssaire pour que ces arguments fonctionnent.

L'article de M.-L. Kakkuri-Knuuttila se concentre sur le répondant en comparant les règles énoncées par Aristote en Topiques, VII 5-7, 10 et ce qui se lit dans Gorgias et Euthydème. Lire ces dialogues avec des lunettes aristotéliciennes permet d'établir le lien entre les règles définitionnelles, les effets épistémiques et les règles stratégiques de l'elenchos. Ainsi l'auteur entend montrer comment Aristote a réalisé un progrès dans l'argument rationnel. H. Fossheim montre que la division est une méthode chez Platon, non pas au sens où elle serait une procédure de progrès intellectuel allant de l'ignorance à la connaissance, mais qu'elle est une manière de présenter de façon systématique des résultats. Le rassemblement et la division sont le point final du dialegesthai, la division se caractérisant comme un exposé de connaissance et non pas comme un moyen pour elle.

Moren S. Thaning examine sur le rapport entre dialectique et dialogue dans le Lysis, et entend montrer ce que L.-A. Dorion soulignait déjà dans son commentaire (GF, 2004) : Platon a construit son dialogue de telle sorte que le lecteur trouve la solution à l'aporie apparente du dialogue. Dialogue protreptique, le Lysis contient les clés de la solution. H. Thesleff se penche sur le Lachès et sa dialectique de recherche commune : le Lachès n'est pas à proprement parler un dialogue socratique, mais pointe déjà vers la dialectique de la République. Charles H. Kahn revient sur «l'importance philosophique du dialogue selon Platon » : en distinguant interprétation littéraire et interprétation philosophique, il affirme que si Platon conçoit la philosophie comme la poursuite d'une vision unifiée, cette unité ne peut pas être figée dans une formulation unique et définitive. Il entend ainsi lire Platon selon le «principe du perspectivisme ». Comparant le Banquet, le Phédon et la République, il montre que la nature des Formes doit être comprise selon la perspective du logos et non pas selon celle de la vision. Quant à la comparaison entre Ménon, Phèdre et République, elle permet d'affirmer que la réminiscence et la vision noétique sont des constructions alternatives du même phénomène : l'accès au domaine des concepts est pour Platon l'accès au domaine de l'Être vrai et de la forme éternelle. Ainsi, les dialogues platoniciens offrent plusieurs formulations de la doctrine des Formes, mais aucune n'est la formulation correcte, encore moins la doctrine des Formes. Il n'y a pas de formulation définitive d'une doctrine des Formes indépendante du contexte.

J. L. Fink se demande «comment Aristote lisait un dialogue platonicien ». Partant d'un passage problématique parce que corrompu de la Poétique, J. L. Fink propose ce qui pourrait être la définition aristotélicienne du dialogue socratique: les écrits socratiques sont un dialogue qui est une partie spécifique de la poésie - un mélange de fiction et de réalisme - qui dépeint l'activité de Socrate - c'est-à-dire ce 
que cet homme a pu faire et dire. S'appuyant sur un passage de la Rhétorique, J. L. Fink affirme que selon Aristote, les dialogues socratiques révèlent tout l'arrière-fond éthique de l'argument dialectique et ont en commun le traitement de la question du genre de vie. Savoir si Socrate parle en son nom ou au nom de Platon importe peu à Aristote, semblable en cela à tous les auteurs antiques, mais non pas aux lecteurs modernes. Aristote en tout état de cause lisait Platon pour y trouver de la philosophie et non pas de la poésie. Comme auteur de dialogue, Aristote se distingue de Platon. Il ne peignait pas de personnage, et s'il se mettait en scène comme principal interlocuteur, le dialogue n'était pas un dialogue par questions/réponses et le répondant y avait un rôle passif. Si la dialectique socratique survit dans plusieurs passages du corpus aristotélicien, ce n'est assurément pas dans les dialogues écrits par Aristote.

Dans sa contribution, V. Politis s'interroge sur ce qu'il y a derrière la question ti esti. V. Politis cherche à savoir ce qui justfie l'association problématique entre la question $t i$ esti et tous les réquisits stricts de la définition. En définitive, ce qui se tient derrière la question $t i$ est $i$ et est donc premier, c'est l'aporie - conclusion plutôt inattendue, en ce qu'elle semble inverser les étapes de la démarche socratique.

H. Ausland se penche sur le sens de l'epagoge: partant de l'idée selon laquelle Aristote l'emploie dans un sens technique, l'auteur examine l'usage socratique de l'epagoge tel qu'on le rencontre chez Platon et Xénophon. Il y a ainsi un sens philosophique et un sens rhétorique ou pédagogique de l'epagoge. Interpréter l'epagoge socratique dans un sens logique révèle donc une influence aristotélicienne, mais on rencontre aussi chez Aristote d'autres usages de l'induction au sens de relation entre exemple et point de vue général. C’est bien ainsi que les néoplatoniciens l'ont comprise, tandis que les péripatéticiens et les modernes ont réduit l'induction à l'inférence inductive.

Dans sa contribution, L.-A. Dorion entend éclairer la définition aristotélicienne de l'elenchos par la sixième définition de la réfutation du Sophiste de Platon qui, bien que nommée réfutation des sophistes, présente toutes les caractéristiques de l'elenchos socratique, dont la subordination de la dimension logique à la dimension morale. Alors que Platon ne reconnaît qu'une forme d'elenchos, l'elenchos dialectique de Socrate, outil de l'éducation morale, Aristote ne souscrit pas à la mission éthique de l'elenchos socratique et en évacue complètement la dimension de la honte et les aspects personnels. Reprenant la thèse exposée dans «La dépersonnalisation aristotélicienne de la dialectique » (Archives de philosophie, 60/4 (1997), p. 597-613), L.-A. Dorion y ajoute un élément déterminant en rendant ici raison de cette profonde modification entre Socrate et Aristote: certes, la dialectique ne doit plus être une activité dangereuse, la pratique de l'elenchos ayant conduit Socrate à la condamnation à mort, mais surtout, Aristote rejette la conception socratique de la vertu-science. L'elenchos aristotélicien n'est pas un fossile de l'elenchos socratique mais a été redéfini de façon seulement logique, entraînant une extension considérable de son domaine d'application. 
Dialectique et réfutation ne concernent pas le soin de l'âme chez Aristote, mais sans doute vaut-il mieux réserver la pratique de l'elenchos aux membres de l'école ce que Platon suggérait déjà dans la République. S’il y a bien une continuité entre Socrate et Aristote, la démoralisation aristotélicienne de la réfutation signifie que pratiquer l'elenchos n'a plus aucun rapport avec l'examen de vie. Or, la dépersonnalisation de la dialectique est déjà à l'œuvre dans certains dialogues de Platon (République, Théétète, Sophiste, Philèbe, Parménide). Ainsi chez Platon, deux conceptions de la dialectique sont présentes et en concurrence. Aristote a très tôt rejeté pour sa part la dimention et le but éthiques de la dialectique socratico-platonicienne.

L'elenchos véritable est selon R. Bolton la réfutation peirastique des Réfutations sophistiques en ce qu'elle montre que la conviction particulière de celui qui est interrogé est en conflit avec des vérités communes. Aristote rejette l'idée selon laquelle la mise en contradiction établit l'ignorance. L'elenchos peirastique établit ainsi la fausseté d'une croyance par rapport à un ensemble d'autres. Or ce qui importe est le degré d'endoxicalité des convictions.

Dans le dernier chapitre, W. Kullman montre comment Aristote s'est progressivement détourné de la dialectique platonicienne en abandonnant la troisième fonction de la dialectique telle qu'elle est caractérisée dans les Topiques, à savoir la recherche des principes des sciences. Cet abandon est lié à ce que les Analytiques présentent comme une nouvelle procédure d'appréhension des principes, à savoir la perception, l'induction et l'expérience - à l'œuvre dans les écrits biologiques. La thèse de W. Kullman est convaincante concernant les écrits biologiques, mais ses affirmations, selon lesquelles les endoxa ne jouent aucun rôle dans le domaine de l'éthique et de la politique, résistent difficilement à la lecture des textes aristotéliciens - par exemple le livre I de l'Éthique à Nicomaque qui, pour définir le bonheur, va opérer tout un mouvement d'examen des endoxa sur l'eudaimonia avant d'en venir à la définition du bonheur à partir de l'ergon de l'homme, conformément à ce qu'Aristote énonce au début du livre VII (1145b2-7).

L'unité de ce livre est assurément le débat dialectique tel qu'il a été inauguré par Socrate et formalisé par Aristote. Et si d'un article à l'autre des thèses différentes, voire contradictoires peuvent être soutenues, tout friand de dialectique ancienne y trouvera de quoi se régaler.

Juliette LEMAIRE

Centre Léon Robin, CNRS/Paris-Sorbonne/ENS Ulm

Kurt LAMPE, The Birth of Hedonism. The Cyrenaic philosophers and Pleasure as a way of life, Princeton University Press 2015, XVII +277 p. ISBN: 9780691161136 (hardback; available also as an e-book)

The monograph by Kurt Lampe is the first systematic attempt in any modern language to deal with the ethics of the Cyrenaics, in particular with their hedonism. The book offers a detailed reconstruction of the ethical doctrines of both 